\title{
Nonalcoholic Fatty Liver Disease and the Need for Action
}

\author{
Ioan Sporea, Roxana Șirli
}

Department of

Gastroenterology and

Hepatology, Victor Babeș

University of Medicine

and Pharmacy, Timișoara,

Romania

\section{Address for correspondence: \\ Roxana Șirli \\ Department of \\ Gastroenterology and \\ Hepatology, Victor Babeș \\ University of Medicine \\ and Pharmacy, Timișoara, \\ Romania \\ roxanasirli@gmail.com}

Received: 21.04.2020

Accepted: 02.05.2020
Nowadays, when the medical world has found the cure of chronic hepatitis $\mathrm{C}$ and achieved the control of chronic hepatitis $B$ infection, two main problems remain for the hepatologists: alcoholic liver disease (ALD) and nonalcoholic fatty liver disease (NAFLD). The problem of ALD remains a social problem, in which the whole community must be involved. However, the problem of NAFLD requires rapid and intensive action.

NAFLD currently affects more than a quarter of the world population [1] and the prevalence is increasing with time, as model estimations show [2]. Overweight and obesity, sedentariness, type 2 diabetes or dyslipidemia are increasing at a global level $[3,4]$. In the last years, many papers and guidelines have been published regarding NAFLD, but now we must consider taking action [5-8].

At this moment, there are two modalities of action. The first one is to screen the general population for fatty liver and the second is to screen only the population at risk: patients with obesity, metabolic syndrome and type 2 diabetes mellitus. What is not very clear, even in the published guidelines, is who should be screened, who should do the screening and which is the best method for screening [5-8]. However, it is clear that the main purpose of NAFLD screening is to identify the patients with at least significant fibrosis, as fibrosis is the main prognosis factor in chronic hepatopathies patients, no matter the etiology [9].

Currently, accurate and simple non-invasive tests to search for significant fibrosis in patients suspected of NAFLD are available. Fibrosis-4 (FIB-4) Index for Liver Fibrosis is a very simple biological test which uses only transaminases serum levels, platelet count and age and can be used by every physician (general practitioners, internal medicine specialists, diabetologists, etc.), with acceptable accuracy to predict significant fibrosis [10]. Other biological tests are more complex and accurate [Enhanced Liver Fibrosis $\left(\mathrm{ELF}^{\mathrm{TM}}\right)$ Test, FibroMax, etc.], while ultrasound based elastography techniques [transient elastography (TE), point Shear Wave Elastography (pSWE) or 2D-SWE] have demonstrated their practical value in many studies [9]. Magnetic Resonance Elastography has became quite common in USA, but even if it is more accurate, it is also much more expensive [9].

Another question is how to select the patients for screening. In our opinion, patients with liver steatosis should be the screening population. In countries where ultrasound is commonly used as a point of care method, a liver ultrasound detecting steatosis is a good argument for further evaluation for fibrosis. If ultrasound is not accessible, a simple biological score for fibrosis can be used to select patients at risk [5-8].

Previous studies have demonstrated that up to $20 \%$ of some categories of patients (with type 2 diabetes or metabolic syndrome) had significant fibrosis $[11,12]$. Screening of these subjects seems mandatory. However, physicians involved directly in the diagnosis, treatment and follow up of these patients (diabetologists, endocrinologists, cardiologists, general practitioners) are not informed enough regarding the "epidemy" of NAFLD in the world $[13,14]$. Thus, we must initiate education programs for physicians and patients at risk. During the scientific meetings of different medical societies, workshops on this topic can be useful.

The recently published paper Identification of patients with advanced fibrosis due to nonalcoholic fatty liver disease: considerations for best practice [15] proposed a simple diagnostic algorithm for clinical practice, including sequential use of noninvasive tests, for early identification of patients at risk of advanced fibrosis due to nonalcoholic steatohepatitis. This algorithm implies primary care screening of patients with one or more features of the metabolic syndrome [16]. It should be performed by means of a non-invasive test which has a high 
negative predictive value for significant fibrosis, such as FIB-4 or nonalcoholic fatty liver disease fibrosis score (NFS). If the patient is found to be at low risk, he/she should be screened again after 3-5 years. If the patient is found to be at high or indeterminate risk for significant fibrosis, he/she should be referred to secondary care for reassessment. Hepatologists should continue with a non-invasive elastographic method (TE, pSWE, 2D-SWE or Magnetic Resonance Elastography) or with a serum-based test, such as FibroTest, FibroMeter or ELF test. Patients found to be at intermediate risk should be proposed for a liver biopsy. Following secondary testing, patients with significant or advanced fibrosis should be monitored every 1-2 years. Patients found to have cirrhosis should be further investigated for its complications and included in the screening program for hepatocellular carcinoma.

Even if a drug treatment is not available for nonalcoholic steatohepatitis, in all patients with NAFLD, very simple measures should be proposed: fight obesity, control diabetes, dyslipidemia and metabolic syndrome. Life-style intervention (diet and exercise) is the most effective way to fight obesity and also has an important role in controlling diabetes and dyslipidemia [5-8]. Bariatric surgery should be considered in morbidly obese patients.

It has also been proven that patients with NAFLD are at a high risk of cardiovascular (CV) diseases, the main cause of death of these patients [17]. NAFLD also has a role in the etiopathogenesis of different $\mathrm{CV}$ manifestations, such as left ventricular dysfunction, atherosclerotic disease, conduction abnormalities and stroke, independent of traditional CV risk factors [18-20]. It has been demonstrated that NAFLD is an independent predictive factor for type 2 diabetes, this being a two-way interaction, since a good control of diabetes and hypertriglyceridemia is improving the prognosis in NAFLD [21]. Furthermore, the severity of liver fibrosis assessed by TE has proved to be an independent factor for survival and CV events [22].

As NASH patients with advanced fibrosis and cirrhosis are at risk for portal hypertension and hepatocellular carcinoma $[23,24]$ they should be included in screening programs for these complications. Not only primary care physicians, but also diabetologists, endocrinologists, internal medicine specialists, cardiologists, involved in screening patients with NAFLD, should be informed about simple non-invasive tests in order to identify advanced fibrosis.

Screening programs should be started before the number of cases with advanced liver fibrosis climbs hhigher. At this moment in many countries one of the main indications for liver transplantation is nonalcoholic steatohepatitis cirrhosis $[25,26]$.

Finally, as NAFLD is now an epidemic disease, we all need to start acting now, not only the hepatologists, but the whole medical community and society.

\section{REFERENCES}

1. Younossi ZM, Koenig AB, Abdelatif D, Fazel Y, Henry L, Wymer M. Global epidemiology of nonalcoholic fatty liver disease-Meta-analytic assessment of prevalence, incidence, and outcomes. Hepatology 2016;64:73-84. doi:10.1002/hep.28431
2. Estes C, Anstee QM, Arias-Loste MT, et al. Modeling NAFLD disease burden in China, France, Germany, Italy, Japan, Spain, United Kingdom, and United States for the period 2016-2030. J Hepatol 2018;69:896-904. doi:10.1016/j.jhep.2018.05.036

3. Saeedi P, Petersohn I, Salpea P, et al; IDF Diabetes Atlas Committee. Global and regional diabetes prevalence estimates for 2019 and projections for 2030 and 2045: Results from the International Diabetes Federation Diabetes Atlas, 9th edition. Diabetes Res Clin Pract 2019;157:107843. doi:10.1016/j.diabres.2019.107843

4. Blüher M. Obesity: global epidemiology and pathogenesis. Nat Rev Endocrinol 2019;15:288-298. doi:10.1038/s41574-019-0176-8

5. European Association for the Study of the Liver (EASL); European Association for the Study of Diabetes (EASD); European Association for the Study of Obesity (EASO). EASL-EASD-EASO Clinical Practice Guidelines for the management of non-alcoholic fatty liver disease. J Hepatol 2016;64:1388-1402. doi:10.1016/j.jhep.2015.11.004

6. Chalasani N, Younossi Z, Lavine JE, et al. The diagnosis and management of nonalcoholic fatty liver disease: Practice guidance from the American Association for the Study of Liver Diseases. Hepatology 2018;67:328-357. doi:10.1002/hep.29367

7. Review Team, LaBrecque DR, Abbas Z, et al; World Gastroenterology Organisation. World Gastroenterology Organisation global guidelines: Nonalcoholic fatty liver disease and nonalcoholic steatohepatitis. J Clin Gastroenterol 2014;48:467-473. doi:10.1097/ MCG.0000000000000116

8. Wong VW, Chan WK, Chitturi S, et al. Asia-Pacific Working Party on Non-alcoholic Fatty Liver Disease guidelines 2017-Part 1: Definition, risk factors and assessment. J Gastroenterol Hepatol 2018;33:70-85. doi:10.1111/jgh.13857

9. European Association for Study of Liver; Asociacion Latinoamericana para el Estudio del Higado. EASL-ALEH Clinical Practice Guidelines: Non-invasive tests for evaluation of liver disease severity and prognosis. J Hepatol 2015;63:237-264. doi:10.1016/j.jhep.2015.04.006

10. McPherson S, Stewart SF, Henderson E, Burt AD, Day CP. Simple noninvasive fibrosis scoring systems can reliably exclude advanced fibrosis in patients with non-alcoholic fatty liver disease. Gut 2010;59:12651269. doi:10.1136/gut.2010.216077

11. Younossi ZM, Tampi RP, Racila A, et al. Economic and Clinical Burden of Non alcoholic Steatohepatitis in Patients With Type 2 Diabetes in the U.S. Diabetes Care 2020;43:283-289. doi:10.2337/dc19-1113

12. Roulot D, Roudot-Thoraval F, NKontchou G, et al. Concomitant screening for liver fibrosis and steatosis in French type 2 diabetic patients using Fibroscan. Liver Int 2017;37:1897-1906. doi:10.1111/liv.13481

13. Polanco-Briceno S, Glass D, Stuntz M, Caze A. Awareness of nonalcoholic steatohepatitis and associated practice patterns of primary care physicians and specialists. BMC Res Notes 2016;9:157. doi:10.1186/ s13104-016-1946-1

14. Patel PJ, Banh X, Horsfall LU, et al. Underappreciation of non-alcoholic fatty liver disease by primary care clinicians: limited awareness of surrogate markers of fibrosis. Intern Med J 2018;48:144-151. doi:10.1111/imj.13667

15. Augustin S, Ahmed A, Alkhouri N, et al. Identification of patients with advanced fibrosis due to nonalcoholic fatty liver disease: considerations for best practice. J Gastrointestin Liver Dis 2020;29(2):235-245. doi:10.15403/jgld-775

16. Alberti KG, Zimmet P, Shaw J. Metabolic syndrome-a new world-wide definition. A Consensus Statement from the International Diabetes Federation. Diabet Med 2006;23:469-480. doi:10.1111/j.14645491.2006.01858.x 
17. Targher G, Day CP, Bonora E. Risk of cardiovascular disease in patient with nonalcoholic fatty liver disease. N Engl J Med 2010;363:1341-1350. doi:10.1056/NEJMra0912063

18. Ma J, Hwang SJ, Pedley A, et al. Bi-directional analysis between fatty liver and cardiovascular disease risk factors. J Hepatol 2017;66:390-397. doi:10.1016/j.jhep.2016.09.022

19. Lonardo A, Ballestri S, Marchesini G, Angulo P, Loria P. Nonalcoholic fatty liver disease: A precursor of the metabolic syndrome. Dig Liver Dis 2015;47:181-190. doi:10.1016/j.dld.2014.09.020

20. Ballestri S, Lonardo A, Bonapace S, Byrne CD, Loria P, Targher G. Risk of cardiovascular, cardiac and arrhythmic complications in patients with non-alcoholic fatty liver disease. World J Gastroenterol 2014;20:1724-1745. doi:10.3748/wjg.v20.i7.1724

21. Byrne CD, Targher G. NAFLD: a multisystem disease. J Hepatol 2015;62 (1 Suppl):S47-S64. doi:10.1016/j.jhep.2014.12.012

22. Shili-Masmoudi S, Wong GL, Hiriart JB, et al. Liver stiffness measurement predicts long-term survival and complications in non- alcoholic fatty liver disease. Liver Int 2020;40:581-589. doi:10.1111/ liv.14301

23. Bertot LC, Jeffrey GP, Wallace M, et al. Nonalcoholic fatty liver disease-related cirrhosis is commonly unrecognized and associated with hepatocellular carcinoma. Hepatol Commun 2017;1:53-60. doi:10.1002/hep4.1018

24. Nagpal SJ, Kabbany MN, Mohamad B, Lopez R, Zein NN, Alkhouri N. Portal hypertension complications are frequently the first presentation of NAFLD in patients undergoing liver transplantation evaluation. Dig Dis Sci 2016;61:2102-2107. doi:10.1007/s10620-016-4124-3

25. Noureddin M, Vipani A, Bresee C, et al. NASH leading cause of liver transplant in women: Updated analysis of indications for liver transplant and ethnic and gender variances. Am J Gastroenterol 2018;113:1649_ 1659. doi:10.1038/s41395-018-0088-6

26. Younossi Z, Stepanova M, Ong JP, et al. Nonalcoholic steatohepatitis is the fastest growing cause of hepatocellular carcinoma in liver transplant candidates. Clin Gastroenterol Hepatol 2019;17:748-755. doi:10.1016/j. cgh.2018.05.057 